\section{Situs inversus en paciente con enfermedad Trofoblastica gestacional d}

\author{
Situs inversus in patients with disease \\ gestational Trophoblastic.

\section{Situs inversus em paciente com doença Trofoblástica gestacional}

Jacqueline Claure Salinas ${ }^{1}$

Skarlen Cespedes Sandoval ${ }^{2}$

\section{Resumen}

Introducción. Situs inversus es un padecimiento poco frecuente en nuestro medio, siendo una alteración congénita del desarrollo embrionario, que se caracteriza por la inversión en espejo de todos los órganos pares. Caso clínico. Paciente de 44 años de edad, internada por el servicio de consulta externa en la Maternidad Germán Urquidi por enfermedad trofoblástica gestacional, anemia moderada. Durante su internación se solicitan estudios complementarios, los cuales en su mayoría reportan como hallazgo esta patología, con inversión de órganos internos del lado contrario. Fue intervenida quirúrgicamente con histerectomía total más salpingooforectomia bilateral, el diagnóstico fue oportuno, para evitar error en los procedimientos posteriores. Conclusión. El diagnóstico temprano es fundamental, dado que el abordaje quirúrgico torácico y abdominal es diferente y determinadas patologías pueden presentarse con clínica inusual. Además, también se puede estudiar la presencia de alteraciones asociadas entre ellas la discinesia ciliar primaria (Síndrome de Kartagener).

Palabras clave: Situs Inversus, Dextrocardia, Enfermedad Trofoblástica Gestacional, Diagnóstico (Fuente: Descriptores en Ciencias de la Salud DeCS).

\section{Abstract}

Introduction. Situs inversus is a rare condition in our midst, being a congenital alteration of embryonic development, that is characterized for mirror inversion of all even organs. Clinical case. 44 years old patient who is hospitalized by the outpatient service in the hospital "Maternidad German Urquidi" for disease gestational trophoblastic, moderate anemia. During his hospitalization, complementary studies are requested which mostly of them report this pathology as a finding, with inversion of internal organs of the opposite side. This patient underwent surgery with total hysterectomy and bilateral salpingoophorectomy, his diagnosis was timely to avoid error in subsequent procedures. Conclusion. Early diagnosis is essential since the thoracic and
Correspondencia a:

${ }^{1}$ Médico especialista, servicio Ginecología y Obstetricia, Hospital Materno Infantil German Urquidi.

Cochabamba - Bolivia.

${ }^{2}$ Médico residente, servicio Ginecología y Obstetricia, Hospital Materno Infantil German Urquidi.

Cochabamba - Bolivia.

Email de contacto:

jaque80273@hotmail.com

scepedessandoval@hotmail.com

Procedencia y arbitraje:

No comisionado, sometido a arbitraje externo

Recibido para publicación: 3 de marzo del 2021 Aceptado para publicación: 20 de mayo del 2021

\section{Citar como:}

Claure Salinas J, Cespedes Sandoval S. Situs inversus en paciente con enfermedad Trofoblastica gestacional. Recisa UNITEPC. 12 de junio de 2021;8(1):57-63.

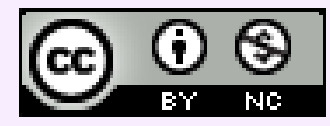

Esta obra está bajo una Licencia Creative Commons Atribución-NoComercial 4.0 Internacional. 
abdominal surgical approach is different. Certain pathologies may occur with unusual clinic. Also it can be studied the presence of associated alteration between them the primary ciliary dyskinesia. (Katagener syndrome).

Keywords: Situs Inversus, Dextrocardia, Gestational Trophoblastic Disease, Diagnosis. (source: Health Sciences Descriptors DeCS).

\section{Resumo}

Introdução: Situs Inversus é uma patologia pouco frequente em nosso meio, sendo uma alteração congênita do desenvolvimento embrionário, que se caracteriza pela inversão de espelho de todos os órgãos pares. Caso clínico: Paciente de 44 anos de idade, internada pelo serviço de consulta externa na Maternidade Germán Urquidi por apresentar enfermidade trofoblástica gestacional, anemia moderada. Durante sua internação, são solicitados estudos complementares, nos quais, em sua maioria, apontam como achado, a referida patologia, com inversão de órgãos internos do lado oposto. Foi realizada intervenção cirúrgica, com histerectomia total mais salpingo-ooforectomia bilateral, com diagnóstico oportuno, para evitar erros nos procedimentos subsequentes. Conclusão: $O$ diagnóstico precoce é fundamental, uma vez que a abordagem cirúrgica torácica e abdominal é diferente. Determinadas patologias podem apresentar sintomas incomuns. Além disso, pode-se estudar a presença de alterações associadas, incluindo discinesia ciliar primária (síndrome de Kartagener).

Palavras-chave: Situs Inversus, Dextrocardia, Doença Trofoblástica Gestacional, Diagnóstico. (fonte: Descritores em Ciências da Saúde: DeCS).

\section{Introducción}

El situs inversus totalis es un hallazgo poco común en nuestro medio, debido a que un porcentaje mínimo de las pacientes lo presenta de 1 de 100000 nacimientos, esta malformación generalmente va asociada a otras malformaciones genéticas. Alteración en el desarrollo embriológico durante la etapa de formación, (tercera semana) como el Síndrome de Kartagener e Ivemark, frecuente en el hombre, pocas presentan cardiopatías asociadas, por lo que el diagnóstico suele realizarse de forma casual $(1,2)$.

El mecanismo exacto por el que ocurre el situs inversus se desconoce; sin embargo, se propone que se debe a un trastorno genético, autosómica recesiva, en el brazo largo del cromosoma 14, que afecta la cascada genética responsable de la diferenciación izquierda-derecha $(3,4)$.

Esta entidad se reconoció en 1643 por Marco Severino, un siglo posterior en 1788, Matthew Baillie describió el cambio total de imagen en espejo de los órganos torácicos y abdominales en el situs inversus. Hay pocos registros de una autopsia en 1774 en México, Marqués de las Amarillas, por Domingo Russi, en la que se describe los hallazgos característicos de la patología $(5,6)$.

Por lo poco común de la patología el objetivo es describir el reporte de un caso acompañado de enfermedad trofoblástica gestacional.

\section{Caso Clínico}

Paciente de 44 años de edad acude en fecha 11 de marzo 2021 por el servicio de consulta externa de la Maternidad Germán Urquidi, referida del hospital Entre Ríos con 
diagnóstico de G5P5, enfermedad trofoblástica gestacional de alto riesgo. Misma que ingresa con diagnósticos de enfermedad trofoblástica gestacional y anemia moderada en tratamiento.

Paciente cuenta con laboratorios del 09 de marzo del 2021 que reporta; GB: 6010 mm³ , Hgb: 9.5 mg/dl, HTO: 29\%, plaquetas: $245000 \mathrm{~mm}^{3}$, segmentados 83\%, TP: 12 segundos, actividad: $100 \%$, INR: 1.0 , Creatinina 0,8 mg/dl, glicemia: $78 \mathrm{mg} / \mathrm{dl}$. Examen general de orina que reporta $\mathrm{pH}$ : 6 , leucocitos: 3 por campo.

Porta ecografía del 24 de febrero del 2021 que reporta mola hidatiforme, quiste simple anexial derecho, útero en AVF mide $16.9 \times 14.8 \times 14.3 \mathrm{~cm}$, en endometrio presencia de masa heterogénea mixta en copo de nieve que mide $10.9 \times 14 \mathrm{~cm}$, en ovario derecho imagen anecogénica circular mide $2.8 \mathrm{~cm}$.

Fracción Beta hCG cuantitativa del 10 de marzo 21 que reporta 1128500 UI/L, tiene antecedente obstétrico G7 P5 A2 C0. No recuerda fecha de última menstruación.

Al examen físico paciente con signos vitales; PA: 100/60 mmhg, FC: 74 lpm, FR: 21 $\mathrm{rpm}, \mathrm{T}^{\circ}: 36.2^{\circ} \mathrm{C}$. Mucosas: ligeramente pálidas. Corazón: rítmico regular normofonético sin ruidos sobreagregados. Pulmones: murmullo vesicular conservado en ambos campos pulmonares. Abdomen: RHA presente normoactivos, blando depresible, poco doloroso a la palpación, aumentado de volumen con altura uterina de $15 \mathrm{~cm}$, tacto bimanual: útero en AVF aproximadamente mide $15 \times 13 \mathrm{~cm}$, anexos: no dolorosos a la movilización, extremidades: tono y trofismo conservado, sin edemas. En servicio se realiza estudios complementarios: perfil tiroideo que reporta $\mathrm{TSH}: 0,1 \mathrm{U} / \mathrm{ml}, \mathrm{T} 3: 4,2$ $\mathrm{mmol} / \mathrm{L}, \mathrm{T} 4: 198 \mathrm{nmol} / \mathrm{L}$, T4 libre:2.0 ng/dl.

Rayos x de tórax que reporta: dextrocardia asociada a leve elevación del hemidiafragma Izquierdo respecto al derecho, por el que no se descarta situs inversus. Ecografía tansvaginal reporta: útero AVF, enfermedad trofoblástica gestacional con interrogación, imagen compleja intrauterina, quiste tecaluteinicos en ovario derecho, ovario izquierdo no visible, posible cistitis, posible gastritis. Ecografía abdominal: reporta situs inversus, dilatación pielocalicial izquierda.

Tomografía abdominopélvica que reporta evidente aumento de tamaño del útero en relación con el diagnóstico de envío bien delimitado sin infiltrar órganos vecinos, solo probable compresión parcial en el tercio distal de uréter izquierdo lo cual condiciona moderada dilatación ureteral y pielocalicial proximal, con contenido en cavidad de tejido isodenso heterogéneo, tórax y abdomen sin otras patologías, todos los órganos torácicos y abdominales se encuentran invertidos compatibles con situs inversus.

Electrocardiograma: ritmo sinusal FC $75 \mathrm{lpm}$, eje $+120^{\circ}$, onda P positiva en AVR —DI bifásica en V5-V6, patrón QR en DI y AVL (situs inversus), se solicita interconsulta con endocrinología por resultados de perfil tiroideo mismo indica hipertiroidismo secundario por lo cual indica danazol $20 \mathrm{mg}$ VO cada día. Valorada por cardiología con riesgo quirúrgico intermedio, paciente recibió dos concentrados de glóbulos rojos previa cirugía, es intervenida quirúrgicamente en fecha 18 de marzo 21. Se realiza histerectomía total abdominal más salpingooforectomia bilateral, hallazgos quirúrgico apéndice cecal, hígado y otras estructuras internas del lado contralateral. Solicitud de laboratorios postquirúrgicos reporta GB: $7660 \mathrm{~mm}^{3}$, Hgb: $10.8 \mathrm{mg} / \mathrm{dl}$, HTO: 33\%, plaquetas: $253000 \mathrm{~mm}^{3}$, fracción Beta hCG cuantitativa: $8248 \mathrm{UI} / \mathrm{L}$. Resultado de patología reporta mola completa, permeación vascular con trombos de trofoblasto atípico. 


\section{Revista Científica de Salud UNITEPC}

ISSN $2520-9825$

La paciente es dada de alta en fecha 26 de marzo del 2021 con resultado fracción Beta hCG cuantitativa $16820 \mathrm{UI} / \mathrm{L}$, antibioticoterapia y tratamiento antiemético, seguimiento por endocrinología y ginecología por consulta externa.

Figura 1: Radiografía anteroposterior de tórax. Se observa la silueta cardiaca localizada en el hemitórax derecho, con el ápex en la misma dirección, se percibe la cámara gástrica $(E)$ con contenido aéreo en la región subdiafragmática derecha, y la sombra hepática $(\mathrm{H})$ izquierda que condiciona la elevación del hemidiafragma ipsilateral.

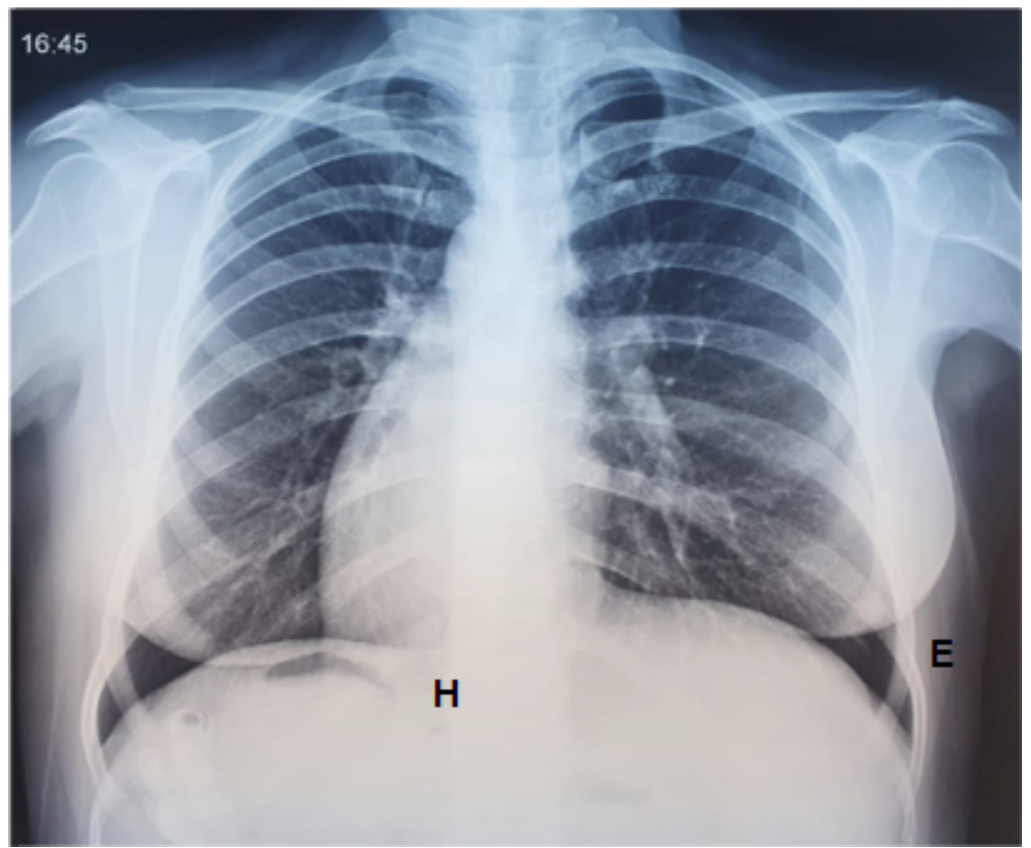

Figura 2: Apéndice cecal en fosa iliaca izquierda.

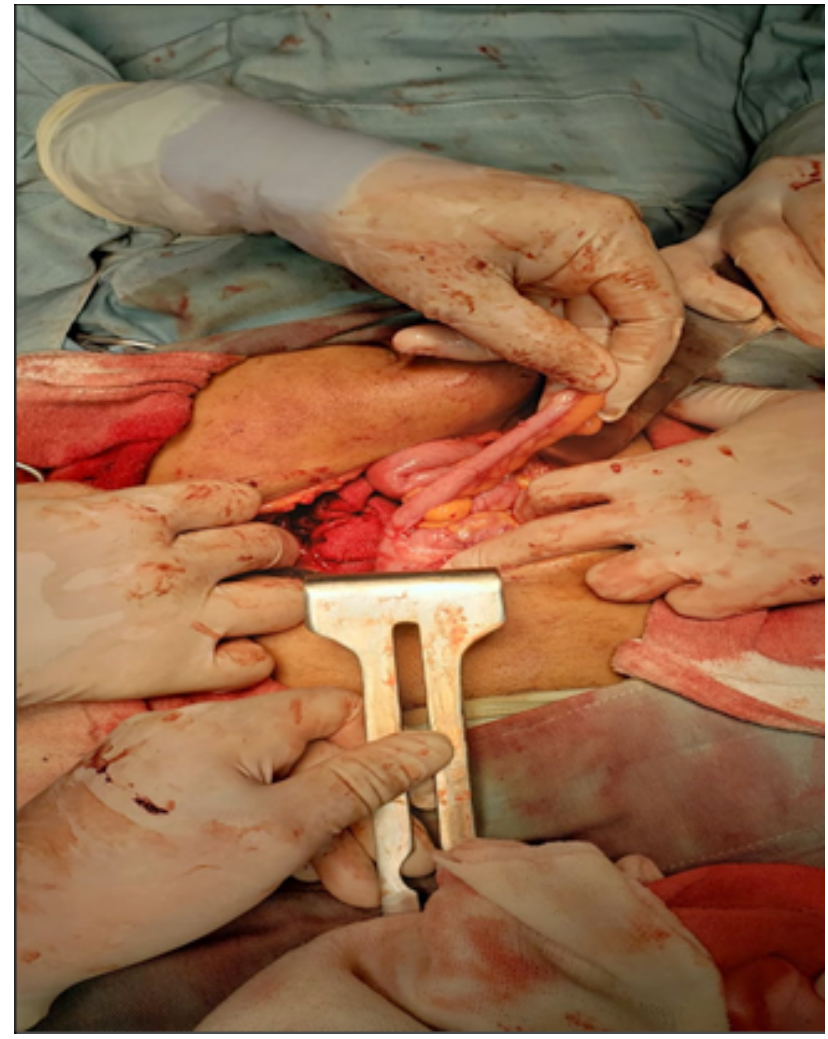


Figura 3: Útero posterior a la histerectomía total, presencia de vesículas en su interior posterior a corte en cara anterior.

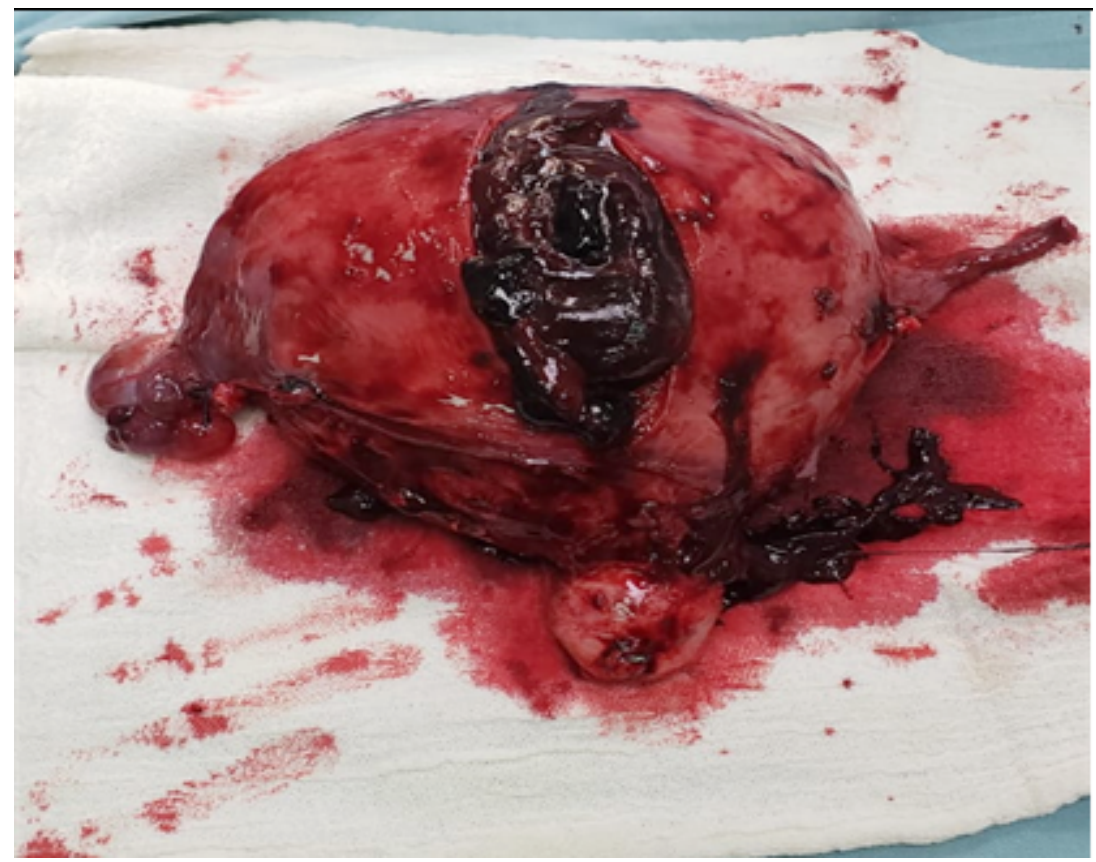

Figura 4: Tomografía axial computarizada compatible con situs inversus.

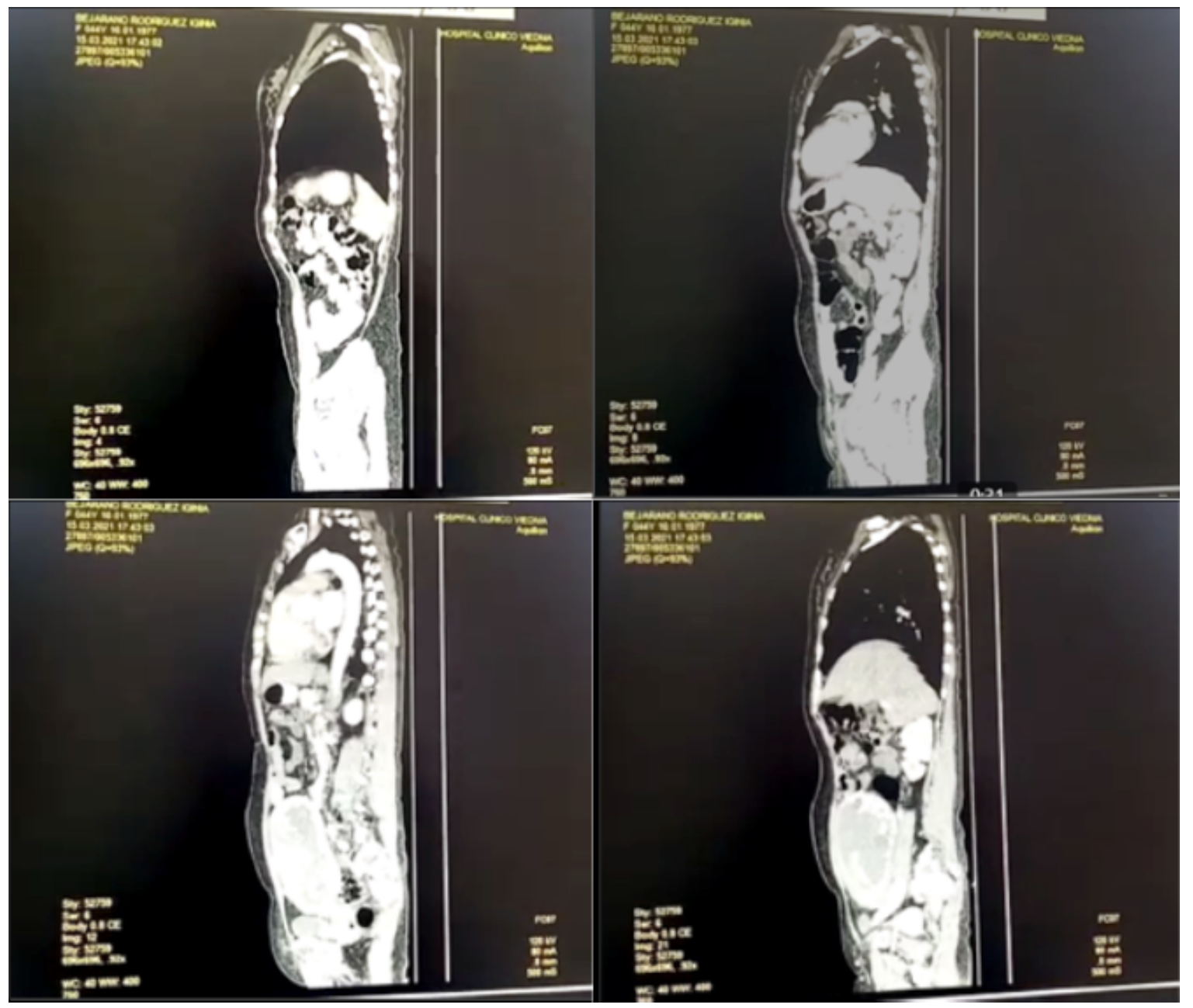




\section{Discusión}

El 99\% de los individuos en etapa embrionaria desarrollan una asimetría izquierda-derecha de los órganos toracoabdominales, denominada situs solitus, en la que encontramos: a la izquierda un pulmón bilobulado, el vértice del corazón, el estómago y el bazo y a la derecha un pulmón trilobulado, vena cava, apéndice y lóbulo mayor del hígado. Con la inversión completa de los órganos lateralizados nos enfrentamos a una situación del situs inversus totalis, un hallazgo que fue descrito por en 1788. Este hecho ocurre en aproximadamente 1/10,000 nacimientos (7-10).

En 5 a 10\% de los pacientes con situs inversus tienen afecciones cardíacas asociadas y malformaciones cardiovasculares que comienzan con síntomas relacionados con estas enfermedades. Los más comunes incluyen la transposición de vasos mayores, un defecto del tabique auricular o ventricular y un retorno venoso anormal. El resto se encuentran asintomáticos y la dextrocardia suele ser un hallazgo incidental en la exploración física o en la radiografía de tórax de rutina como ocurre en los casos clínicos presentados (11).

El situs inversus totalis al ser un hallazgo casual, ya que la mayoría de los pacientes son asintomáticos, por lo que es diagnosticado por estudios complementarios de forma incidental.

En la actualidad se han adjuntado varios estudios asociados a su detección en etapas tempranas estos son: tonos cardíacos en el hemitórax derecho, un electrocardiograma sugestivo de dextrocardia donde se encuentra ejes de $P$ y QRS entre $+120^{\circ}$ y $+150^{\circ}$, voltaje atenuado del QRS en precordiales izquierdas y ondas T negativas en precordiales derechas, además de la radiografía de tórax, ecografía abdominal y ecocardiografía para detectar posibles cardiopatías.

Debido a que el hallazgo es tardío y el diagnóstico temprano es fundamental más aún cuando el paciente es sometido a procedimiento quirúrgico, además que la clínica siempre será opuesta, por lo tanto, el realizar un estudio oportuno mejora calidad en la atención y mejor pronóstico de vida.

\section{Conflictos de Intereses}

Los autores declaramos no tener conflictos de interés para el presente estudio.

\section{Referencias bibliográficas}

1. Gort Hernández M. Situs inversus totalis: presentación de un caso. Rev Cienc Médicas Pinar Río. marzo de 2010;14(1):250-5.

2. Reales $\bigvee$ de JA, Gallego GCÁ, Espitia NCÁ, Coley AA, Suárez OGÁ, Reales $\vee$ de $\mathrm{JA}$, et al. Situs inversus totalis: revisión de tema con aproximación a la Genética y reporte de casos. Rev Colomb Cardiol. 2017;24(1):40-7.

3. Brito Velasquez M, Narvaez R J. Situs inversus totalis. Reporte de un caso. Cienc E Investig Medico Estud Latinoam CIMEL. 2014;19(1):38-40.

4. Melchor-González JM, Pérez-García R, Torres-Vista M, Rodríguez-Brambila VR. Situs inversus. Reporte de dos casos. Cir Cir. 2000;68(2):72-5.

5. Spoon JM. Situs inversus totalis. Neonatal Netw NN. 2001;20(1):59-63.

6. Gentile BA, Tighe DA. Situs Inversus Totalis. N Engl J Med. 2019;380(24):e45. 
7. Aylsworth AS. Clinical aspects of defects in the determination of laterality. Am J Med Genet. 2001;101(4):345-55.

8. Kosaki K, Casey B. Genetics of human left-right axis malformations. Semin Cell Dev Biol. 1998;9(1):89-99.

9. Marta MJ, Falcão LM, Saavedra JA, Ravara L. A case of complete situs inversus. Rev Port Cardiol Orgao Of Soc Port Cardiol Port J Cardiol Off J Port Soc Cardiol. 2003;22(1):91-104.

10. Paublo M M, Bustos V JC, Ramírez H P. Diagnóstico prenatal de situs inversus totalis. Rev Chil Obstet Ginecol. 2002;67(6):494-7.

11. Juncos C M, Ros F MA, Maravall LI M, Álvarez-Pitti J. Situs inversus totalis: A propósito de 2 casos clínicos. Rev Chil Pediatría. 2014;85(3):344-50. 